\title{
CANGAROO-III OBSERVATION OF TeV GAMMA RAYS FROM THE UNIDENTIFIED GAMMA-RAY SOURCE HESS J1614-518
}

\author{
T. Mizukami ${ }^{1}$, H. Kubo ${ }^{1}$, T. Yoshida $^{2}$, T. Nakamori ${ }^{3}$, R. Enomoto ${ }^{4}$, T. Tanimori ${ }^{1}$, M. Akimoto ${ }^{5}$, G. V. Bicknell $^{6}$, \\ R. W. Clay ${ }^{7}$, P. G. Edwards ${ }^{8}$, S. Gunit ${ }^{9}$, S. Hara ${ }^{10}$, T. Hara ${ }^{10}$, S. Hayashi ${ }^{11}$, H. Ishioka ${ }^{5}$, S. Kabuki ${ }^{1}$, F. KaJino ${ }^{11}$, \\ H. Katagiri ${ }^{12}$, A. KawachI ${ }^{5}$, T. Kifune ${ }^{4}$, R. Kiuchi ${ }^{13}$, T. Kunisawa ${ }^{4}$, J. Kushida ${ }^{5}$, T. Matoba ${ }^{2}$, Y. Matsubara ${ }^{14}$, \\ I. Matsuzawa ${ }^{5}$, Y. Mizumura ${ }^{5}$, Y. Mizumoto ${ }^{15}$, M. Mori ${ }^{16}$, H. Muraishi $^{17}$, T. Naito ${ }^{10}$, K. NaKayama ${ }^{4}$, K. Nishijima ${ }^{5}$, \\ M. Ohishi ${ }^{4}$, Y. Otake ${ }^{9}$, S. Ryoki ${ }^{4}$, K. Saito ${ }^{5}$, Y. Sakamoto ${ }^{5}$, V. Stamatescu ${ }^{7}$, T. SuZuki ${ }^{2}$, D. L. Swaby ${ }^{7}$, G. Thornton ${ }^{7}$,

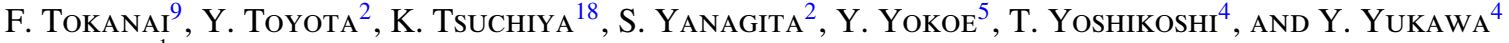 \\ ${ }^{1}$ Department of Physics, Graduate School of Science, Kyoto University, Sakyo-ku, Kyoto 606-8502, Japan; \\ mizukami@cr.scphys.kyoto-u.ac.jp, kubo@cr.scphys.kyoto-u.ac.jp \\ ${ }^{2}$ Faculty of Science, Ibaraki University, Mito, Ibaraki 310-8512, Japan \\ ${ }^{3}$ Graduate School of Advanced Science and Engineering, Faculty of Science and Engineering, \\ Waseda University, 3-4-1 Ohkubo, Shinjuku, Tokyo 169-0072, Japan \\ ${ }^{4}$ Institute for Cosmic Ray Research, University of Tokyo, Kashiwa, Chiba 277-8582, Japan \\ ${ }^{5}$ Department of Physics, Tokai University, Hiratsuka, Kanagawa 259-1292, Japan \\ ${ }^{6}$ Research School of Astronomy and Astrophysics, Australian National University, ACT 2611, Australia \\ ${ }^{7}$ School of Chemistry and Physics, University of Adelaide, SA 5005, Australia \\ ${ }^{8}$ CSIRO Astronomy and Space Science, Australia Telescope National Facility, Epping, NSW 2121, Australia \\ ${ }^{9}$ Department of Physics, Yamagata University, Yamagata, Yamagata 990-8560, Japan \\ ${ }^{10}$ Faculty of Management Information, Yamanashi Gakuin University, Kofu, Yamanashi 400-8575, Japan \\ ${ }^{11}$ Department of Physics, Konan University, Kobe, Hyogo 658-8501, Japan \\ 12 Department of Physical Science, Hiroshima University, Higashi-Hiroshima, Hiroshima 739-8526, Japan \\ 13 Institute of Particle and Nuclear Studies, High Energy Accelerator Research Organization, 1-1 Oho, Tsukuba, Ibaraki, 305-0801, Japan \\ ${ }^{14}$ Solar-Terrestrial Environment Laboratory, Nagoya University, Nagoya, Aichi 464-8602, Japan \\ 15 National Astronomical Observatory of Japan, Mitaka, Tokyo 181-8588, Japan \\ ${ }^{16}$ Department of Physics, College of Science and Engineering, Ritsumeikan University, 1-1-1 Nojihigashi, Kusatsu, Shiga, 525-8577, Japan \\ ${ }^{17}$ School of Allied Health Sciences, Kitasato University, Sagamihara, Kanagawa 228-8555, Japan \\ 18 National Research Institute of Police Science, Kashiwa, Chiba 277-0882, Japan \\ Received 2010 March 15; accepted 2011 July 9; published 2011 October 3
}

\begin{abstract}
We report the detection, with the CANGAROO-III imaging atmospheric Cherenkov telescope array, of a very high energy gamma-ray signal from the unidentified gamma-ray source HESS J1614-518, which was discovered in the H.E.S.S. Galactic plane survey. Diffuse gamma-ray emission was detected above $760 \mathrm{GeV}$ at the $8.9 \sigma$ level during an effective exposure of $54 \mathrm{hr}$ from 2008 May to August. The spectrum can be represented by a power law: $\left(8.2 \pm 2.2_{\text {stat }} \pm 2.5_{\text {sys }}\right) \times 10^{-12} \times(E / 1 \mathrm{TeV})^{-\gamma} \mathrm{cm}^{-2} \mathrm{~s}^{-1} \mathrm{TeV}^{-1}$ with a photon index $\gamma$ of $2.4 \pm 0.3_{\text {stat }} \pm 0.2_{\text {sys }}$, which is compatible with that of the H.E.S.S. observations. By combining our result with multiwavelength data, we discuss the possible counterparts for HESS J1614-518 and consider radiation mechanisms based on hadronic and leptonic processes for a supernova remnant (SNR), stellar winds from massive stars, and a pulsar wind nebula $(\mathrm{PWN})$. Although a leptonic origin from a PWN driven by an unknown pulsar remains possible, hadronic-origin emission from an unknown SNR is preferred.
\end{abstract}

Key words: gamma rays: ISM - ISM: individual objects (HESS J1614-518)

Online-only material: color figures

\section{INTRODUCTION}

Recent progress with Imaging Air Cherenkov Telescopes (IACTs) is enabling the exploration of sites of cosmic-ray acceleration in our galaxy. Very high energy (VHE) gamma rays are produced by the decay of neutral pions which arise from interactions between the accelerated protons and interstellar matter, or by inverse Compton (IC) scattering and Bremsstrahlung of high-energy electrons. For example, VHE gamma rays have been detected from young supernova remnants (SNRs) such as RX J1713.7-3946 (Muraishi et al. 2000; Enomoto et al. 2002b; Aharonian et al. 2006c), RX J0852.0-4622 (Katagiri et al. 2005; Aharonian et al. 2005e; Enomoto et al. 2006b), and RCW86 (Watanabe et al. 2003; Aharonian et al. 2009), which show possible evidence of cosmic-ray acceleration (e.g., Enomoto et al. 2002b; Malkov et al. 2005; Uchiyama et al. 2007; Tanaka et al. 2008; Yamazaki et al. 2009). In addition, detections of VHE gamma rays from pulsar wind nebulae (PWNe) such as the Crab nebula (Weekes et al. 1989) and Vela X nebula (Aharonian et al. 2006b; Enomoto et al. 2006a) have shown that PWNe also play an important role in particle acceleration in the Galaxy. Recently, VHE gamma-ray emission related to massive stars such as the X-ray binary systems PSR B1259-63 (Aharonian et al. 2005d) and LS I + 61303 (Albert et al. 2006), the young open stellar clusters Cyg OB2 (Aharonian et al. 2002), Westerlund 2 (Aharonian et al. 2007), and Westerlund 1 (Ohm et al. 2010) have also been reported. Moreover, the Galactic plane survey performed by the H.E.S.S. observatory (Aharonian et al. 2005c, 2006a) discovered 17 unidentified VHE gamma-ray sources, including HESS J1614-518. Today, unidentified sources are the largest class of the 123 discovered VHE gamma-ray sources, most of which are located in the Galactic plane (e.g., Aharonian et al. 2005a; Abdo et al. 2007; Aharonian et al. 2008; the TeVCAT catalog, TeVCAT ver.3.400 (2011), is a useful up-to-date, 
online resource). In general, the lack of non-thermal electromagnetic radiation from the radio to the X-ray bands may be evidence of hadron acceleration because the IC scenario requires a lower magnetic field than the typical interstellar magnetic field intensity of a few $\mu \mathrm{G}$. Revealing the possible radiation mechanism(s) of each unidentified source is therefore important for identifying the origin(s) of cosmic rays.

H.E.S.S. reported that HESS J1614-518 had a high flux level, $25 \%$ of the Crab nebula, above $200 \mathrm{GeV}$ with a photon index of 2.4, and an elliptical morphology with a semimajor axis of $14 \pm 1$ arcmin and a semiminor axis of $9 \pm 1$ arcmin (Aharonian et al. 2006a). The peak position has an offset of 8.7 arcmin to the northeast from the central position. Landi et al. (2007) and Rowell et al. (2008) pointed out that HESS J1614-518 may be associated with the 40 Myr old young open star cluster Pismis 22 (Piatti et al. 2000) which is located within the VHE gamma-ray emission region at a distance of $1.0 \pm 0.4 \mathrm{kpc}$ and has sufficient luminosity to produce the observed gamma-ray luminosity, assuming $20 \%$ energy conversion from the stellar winds of 10 B-type stars. However, there are several issues in identifying HESS J1614-518 with Pismis 22 since the size of Pismis 22, 2.0 arcmin in diameter, is one order of magnitude smaller than the VHE gamma-ray emission size and the location has a 12 arcmin offset from the VHE gamma-ray emission peak. In addition, there has been no detailed discussion of the radiation mechanism.

The X-ray satellite Suzaku observed this region with the X-ray imaging spectrometer (XIS) and found three X-ray sources in their follow-up observation in 2006 (Matsumoto et al. 2008). One of these, called Suzaku source A, is located very close to the VHE gamma-ray peak position with an offset of 0.8 arcmin. The spectrum is well fitted by a single powerlaw model with a photon index of $1.73_{-0.30}^{+0.33}$ and a hydrogen equivalent column density of $1.21_{-0.41}^{+0.50} \times 10^{22} \mathrm{~cm}^{-2}$. The distance to Suzaku source A is approximately $10 \mathrm{kpc}$, which was derived from the hydrogen equivalent column density using the total Galactic H i column density toward HESS J1614-518 of $\sim 2.2 \times 10^{22} \mathrm{~cm}^{-2}$ (Dickey \& Lockman 1990). The size of the X-ray emission region is slightly larger than the Suzaku point-spread function (PSF) of 1.8 arcmin and smaller than the size of the VHE gamma-ray region. This difference is seen in PWNe such as HESS J1825-137 (Aharonian et al. 2006d; Uchiyama et al. 2009) and Vela X (Markwardt \& Ogelman 1995; Aharonian et al. 2006b) and could be explained by the difference between the synchrotron cooling time of the electrons that radiate $\mathrm{X}$-rays and those that produce $\mathrm{TeV}$ gamma rays. Electrons with an energy of $100 \mathrm{TeV}$ radiate $\mathrm{X}$-rays and immediately lose their energy by synchrotron cooling (e.g., the energy-loss timescale is $\sim 10^{2}$ yr assuming a magnetic field of $20 \mu \mathrm{G}$; Sturner et al. 1997), while electrons with an energy of $1 \mathrm{TeV}$, which are responsible for the VHE gamma-ray emission through IC scattering, are more slowly cooled by synchrotron radiation (e.g., the energy-loss timescale is $\sim 10^{4}$ yr assuming the same parameters as above) and can travel farther from their source. However, the ratio between the observed VHE gammaray and X-ray fluxes, $F(1-10 \mathrm{TeV}) / F(2-10 \mathrm{keV})$ of $\sim 34$, is much larger than those of known PWNe-2.6 $\times 10^{-3}, 0.7$, and 1.5 for the Crab, MSH 15-52, and Vela X, respectively (Gaensler et al. 1999; Willingale et al. 2001; Gaensler et al. 2002; Dodson et al. 2003; Aharonian et al. 2004, 2005b, 2006b; Manzali et al. 2007; Nakamori et al. 2008). Nevertheless, recent studies of HESS J1640-465 (Funk et al. 2007) and HESS J1804-216 (Higashi et al. 2008) claim that this large ratio can be explained by a time-evolving electron injection model, in which the number of electrons injected into space by the pulsar decreases proportionally to the spin-down of the pulsar. On the other hand, this large ratio is also expected in an old SNR with an age of $\sim 10^{5} \mathrm{yr}$ because of the difference between the cooling times of electrons and protons (Yamazaki et al. 2006). We therefore discuss both a PWN scenario and an SNR scenario in this paper.

Suzaku source B is positioned toward the center of HESS J1614-518 and is coincident with the position of Pismis 22. Since the hydrogen equivalent column density derived from the Suzaku spectrum is $(1.1 \pm 0.21) \times 10^{22} \mathrm{~cm}^{-2}$, which is comparable with that of Suzaku source A, Suzaku source B may lie at a similar distance to Suzaku source A. This source has a non-thermal X-ray emission with a photon index of 3.19 \pm 0.32 . This soft index and X-ray luminosities of $7.7 \times 10^{34} \mathrm{erg} \mathrm{s}^{-1}$ and $4.5 \times 10^{35} \mathrm{erg} \mathrm{s}^{-1}$ in the $2-10 \mathrm{keV}$ and $0.5-10 \mathrm{keV}$ ranges, respectively, assuming a distance of $10 \mathrm{kpc}$, are typical values for an anomalous X-ray pulsar (AXP; Fahlman \& Gregory 1981; Kuiper et al. 2006). The possible existence of the AXP also suggests that this source may be an SNR, since AXPs are usually associated with SNRs, e.g., 1E 2259+586 with CTB 109 (Fahlman \& Gregory 1981) and 1E 1841-045 with Kes 73 (Vasisht \& Gotthelf 1997). Since the position of this source is coincident with Pismis 22, there is a possibility that this emission originates from the stellar winds from the stellar cluster. Non-thermal X-ray emission from a stellar cluster was reported from Westerlund 1 (Muno et al. 2006), and TeV gamma rays were recently detected from this object (Ohm et al. 2009). However, this positional correlation may be only a chance coincidence since the estimated distances to Suzaku source B and Pismis 22 are different by an order of magnitude. Although Suzaku source B might be marginally extended, it is difficult to quantitatively estimate the spatial extension with the Suzaku PSF of 1.8 arcmin. If Suzaku source B is actually extended, additional scenarios besides an SNR could be considered, e.g., a PWN from a pulsar/AXP as discussed in Matsumoto et al. (2008), or emission from the unresolved hot stars in Pismis 22.

The other source, Suzaku source C, is a late B-type star as described in Matsumoto et al. (2008), and thus is not a possible counterpart of HESS J1614-518.

Swift observed this region with the X-Ray Telescope (XRT) and found six X-ray sources (hereafter Swift sources 1-6; Landi et al. 2006, 2007). All these sources were point-like and no diffuse emission was found. Two sources, Swift sources 1 and 4, are located within the field of view (FOV) of the Suzaku observation. Swift source 1 is located close to Pismis 22 with an offset of 42 arcsec. This source is also coincident with Suzaku source B. Swift source 4 is coincident with Suzaku source C. Swift sources 1, 2, 3, and 5 are probably stars, while the nature of Swift sources 4 and 6 were not identified, probably due to the poor statistics. Although Suzaku source A was located in the FOV of the Swift XRT, it was not detected with Swift probably due to the limited exposure time $(\sim 1700 \mathrm{~s})$ and/or the small effective area.

The Fermi-LAT Collaboration (Abdo et al. 2010a) reported the detection of gamma rays in the $100 \mathrm{MeV}$ to $100 \mathrm{GeV}$ band from 1FGL J1614.7-5138c positioned 2.7 arcmin away from the peak position of the VHE gamma-ray emission. In the radio band, no counterpart has been found in the HESS J1614-518 region; there is no enhancement in the $843 \mathrm{MHz}$ band, where the rms noise level is $\sim 2 \mathrm{mJy} \operatorname{arcmin}^{-2}$ (Bock et al. 1999; Murphy et al. 2007). In this paper, we present TeV gamma-ray 
observations of HESS J1614-518 with the CANGAROO-III telescopes and discuss the possible counterpart and the radiation mechanism by considering multiwavelength observations.

\section{OBSERVATIONS}

CANGAROO-III is an array of four IACTs (T1, T2, T3, and T4), located at Woomera, South Australia (136 $47 \mathrm{E}, 31^{\circ} 06 \mathrm{~S}$, $160 \mathrm{~m}$ a.s.l.; Enomoto et al. 2002a). The oldest telescope, T1, which was the CANGAROO-II telescope, has not been in use since 2004 due to its smaller FOV and higher energy threshold. Each telescope has a $10 \mathrm{~m}$ diameter reflector which consists of 114 segmented fiber reinforced plastic spherical mirrors mounted on a parabolic frame (Kawachi et al. 2001). The imaging camera system consists of 427 photomultipliers (PMTs) and has an FOV of 4.0 deg (Kabuki et al. 2003). The PMT signals are digitized by charge analog-to-digital converters (ADCs) and multi-hit time-to-digital converters (TDCs; Kubo et al. 2001). The observations were carried out from 2008 May to August using a wobble mode in which the pointing position was shifted both in declination and right ascension between $\pm 0.5 \mathrm{deg}$ from the target position every 20 minutes (Nakamori et al. 2008). The target position was (R.A., decl. [J2000]) = $(243.579,-51.820)$ which is the center of the source position reported by H.E.S.S.

The light-collecting efficiency, including the reflectivity of the mirror segments and the light guides, and the quantum efficiency of PMTs, was monitored by a muon-ring analysis with individual trigger data taken in the same period (Enomoto et al. 2006a). The average quantity of light per unit arc length of muon rings is approximately proportional to the light-collecting efficiency. We also did not use the second oldest telescope, T2, since it was very difficult to calibrate with muon-ring data to calculate the efficiency at this time due to the deterioration of mirror reflectivity (Enomoto et al. 2009). The two telescopes T3 and T4 were used. From the muon-ring analysis for the data taken in this period, the light-collecting efficiency of each telescope, which is used in the Monte Carlo simulations, with respect to the original mirror production time, was 0.58 and 0.50 for $\mathrm{T} 3$ and $\mathrm{T} 4$, respectively. We reject the data for which the average trigger rate over a 1 minute period was under $5 \mathrm{~Hz}$ to remove data taken in cloudy conditions. The effective exposure time amounts to $53.6 \mathrm{hr}$ and the energy threshold was $760 \mathrm{GeV}$.

\section{ANALYSIS}

The standard analysis of the CANGAROO-III collaboration (Enomoto et al. 2006a; Kabuki et al. 2007) was applied to the data. The calibrations for the cameras and ADCs were carried out daily using LEDs. After calibration, the recorded charges of each pixel in the camera were converted to the number of photoelectrons. Time-walk corrections for TDC data were carried out using data taken by changing the luminosity of the LEDs. After calibration, every shower image was cleaned through the following criteria. Only pixels that had $\geqslant 5.0$ photoelectrons were used as "hit pixels." Clusters of five or more adjacent hit pixels with arrival times within $30 \mathrm{~ns}$ of the average hit time of all pixels were recognized as a shower cluster. Before calculating image moments - the "Hillas parameters" (Hillas 1985) — we applied an "edge cut" to the data (Enomoto et al. $2006 \mathrm{~b}$ ) and rejected events having hit pixels in the outermost layer of the camera. The orientation angles were determined by minimizing the sum of the squared widths with a constraint given by the distance predicted by Monte Carlo simulations.

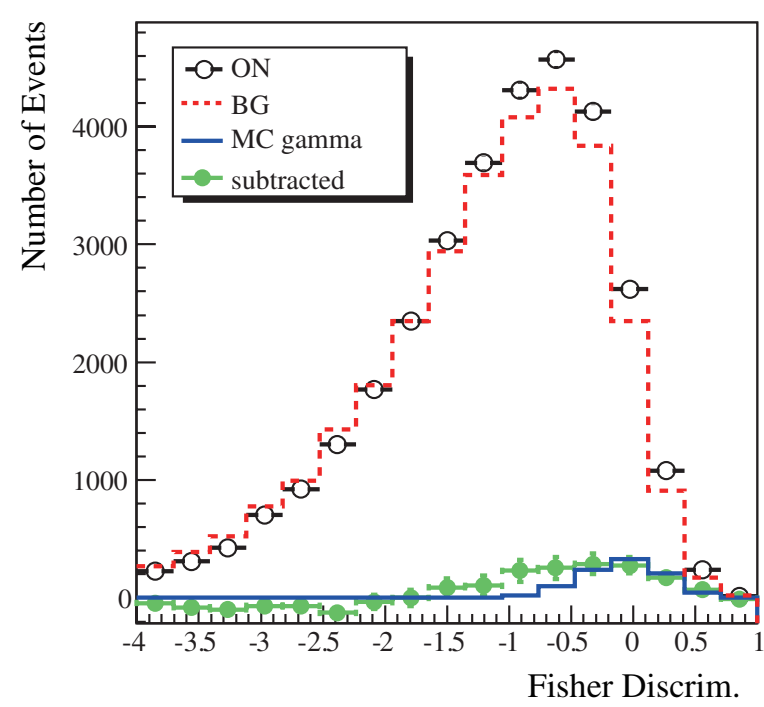

Figure 1. FD distribution. The black circles show the FD obtained from the ON source region, $\theta^{2} \leqslant 0.2 \mathrm{deg}^{2}$. The red and blue lines are the background and gamma-ray component estimated by the fit procedure described in the text. The green circles are obtained by the subtraction of the background from the ON source region.

(A color version of this figure is available in the online journal.)

In order to derive the gamma-ray likeliness, we used the Fisher Discriminant method (Fisher 1936; Enomoto et al. 2006a). The input parameters were

$$
\vec{P}=\left(W_{3}, W_{4}, L_{3}, L_{4}\right),
$$

where $W_{3}, W_{4}, L_{3}$, and $L_{4}$ are energy-corrected widths and lengths for T3 and T4 camera images. The Fisher discriminant (hereafter FD) is defined as FD $=\vec{\alpha} \cdot \vec{P}$, where $\vec{\alpha}$ is a set of coefficients mathematically determined in order to maximize the separation between two FD distributions for gamma rays and hadrons.

To calculate the background, we selected a ring region around the target, $0.3 \mathrm{deg}^{2} \leqslant \theta^{2} \leqslant 0.5 \mathrm{deg}^{2}$, where $\theta$ is the angular distance to the center of HESS J1614-518 (Higashi et al. 2008). We obtained the FD distributions of hadrons $F_{b}$ from this region and gamma rays $F_{g}$ from Monte Carlo simulations. Finally, we were able to fit the FD distribution of the events from the target with a linear combination of these two components. The observed FD distributions $F$ were represented by

$$
F=\beta F_{g}+(1-\beta) F_{b}
$$

where $\beta$ is the ratio of gamma-ray events to the total number of events. Here $\beta$ is a fitting parameter and the obtained FD distributions are shown in Figure 1.

\section{RESULTS}

The obtained $\theta^{2}$ plot is shown in Figure 2 with the PSF of our telescopes. Above $760 \mathrm{GeV}$ we detected $950 \pm 107$ excess events within $\theta^{2} \leqslant 0.2 \mathrm{deg}^{2}$. The morphology of gammaray-like events, obtained from a Gaussian smoothing with the CANGAROO-III PSF of $0.24 \mathrm{deg}$, is shown in Figure 3. The extent of the VHE gamma-ray emission was estimated by a two-dimensional Gaussian fit on our excess map. The obtained standard deviation was $0.44 \pm 0.03 \mathrm{deg}$ which is broader than the CANGAROO-III PSF. The centroid position was determined to be (R.A., decl. [J2000]) $=(243.634,-51.950)$. 


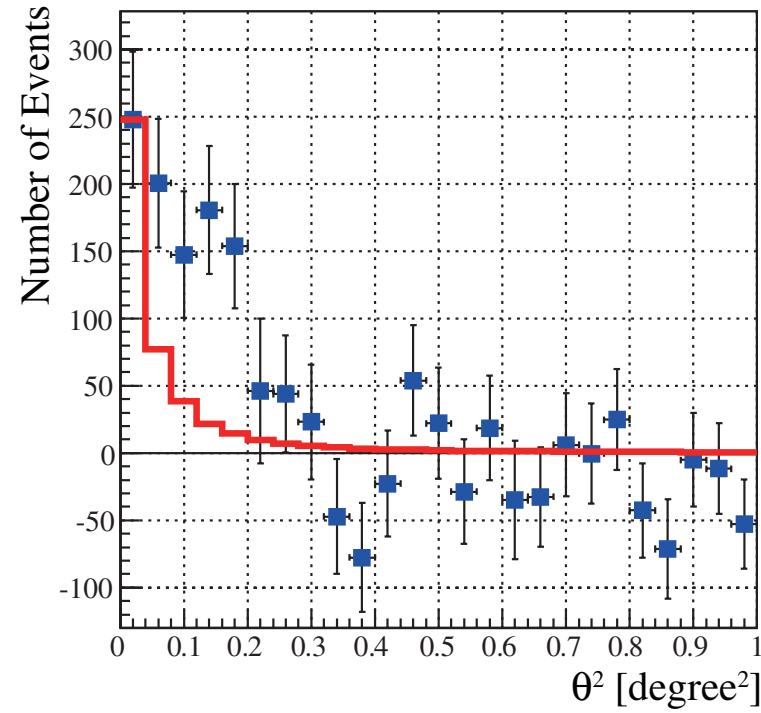

Figure 2. $\theta^{2}$ plot, where $\theta^{2}=0$ corresponds to the fitted center of gravity of HESS J1614-518 from H.E.S.S. (Aharonian et al. 2006a). The blue data points represent the excess events in each $\theta^{2}$ bin and the red solid line represents our PSF derived from the Monte Carlo simulation.

(A color version of this figure is available in the online journal.)

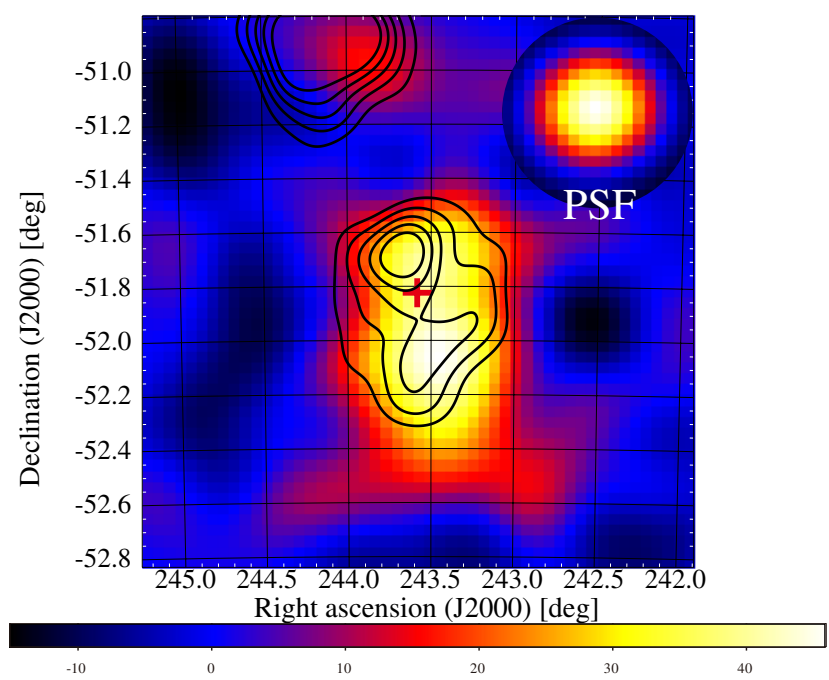

Figure 3. Morphology of gamma-ray-like events. The number of excess events per $0.04 \times 0.04$ cell is smoothed by a Gaussian with $\sigma=0.24 \mathrm{deg}$, which is the CANGAROO-III PSF, and plotted in equatorial coordinates. The black solid contours show the VHE gamma-ray emission seen by H.E.S.S. Lines correspond to 20, 30, 40, 50, and 60 gamma-ray counts. The red cross shows the H.E.S.S. center of gravity of HESS J1614-518 (Aharonian et al. 2006a).

(A color version of this figure is available in the online journal.)

The offset from the best-fit position reported by H.E.S.S. is $(\triangle$ R.A., $\triangle$ decl. $)=(0.055 \pm 0.018,-0.130 \pm 0.033)$. The offset is not significant compared with our PSF. A systematic difference due to the difference in energy thresholds between H.E.S.S. and CANGAROO-III may also contribute to this offset. The reconstructed VHE gamma-ray differential flux is shown in Figure 4 together with the H.E.S.S. measurements. The spectrum is compatible with a single power law: $(8.2 \pm$ $\left.2.2_{\text {stat }} \pm 2.5_{\text {sys }}\right) \times 10^{-12} \times(E / 1 \mathrm{TeV})^{-\gamma} \mathrm{cm}^{-2} \mathrm{~s}^{-1} \mathrm{TeV}^{-1}$ with a photon index $\gamma$ of $2.4 \pm 0.3_{\text {stat }} \pm 0.2_{\text {sys. }}$. The relevant systematic errors are due to the atmospheric transparency, night-sky background fluctuations, uniformity of camera pixels,

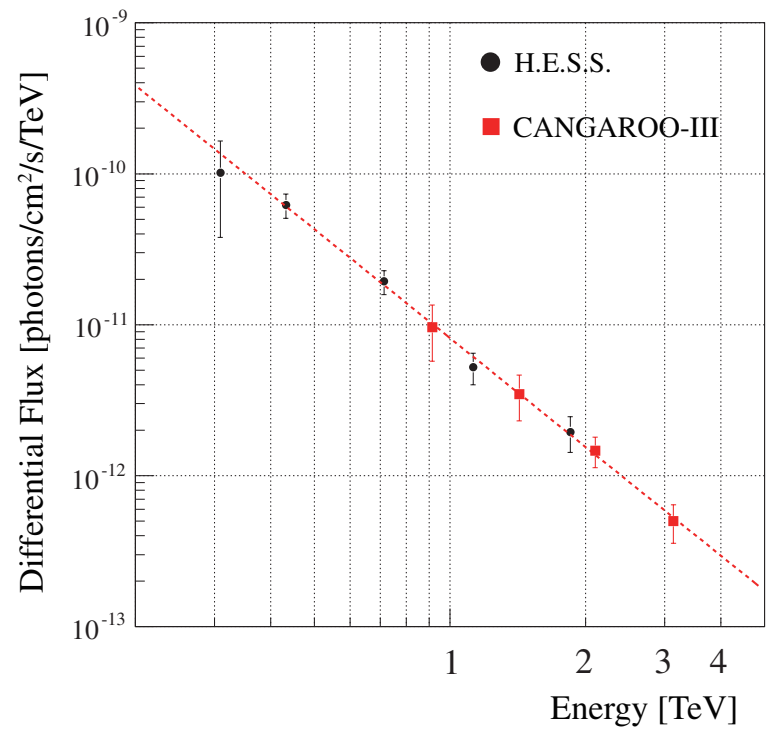

Figure 4. Differential flux of HESS J1614-518. Squares and circles show the CANGAROO-III and the H.E.S.S. data points, respectively. The best-fit power law from this work is shown by the dotted line.

(A color version of this figure is available in the online journal.)

and light-collecting efficiencies. In addition, to estimate the systematic error due to the size of signal integration region, we changed the region from $\theta^{2}<0.14 \mathrm{deg}^{2}$ to $0.30 \mathrm{deg}^{2}$, which was included in the systematic error. For comparison with the ring-region background, we took background events from opposite positions of HESS J1614-518 observations in the wobble mode, and then obtained a differential flux of $\left(6.4 \pm 2.0_{\text {stat }} \pm 2.4_{\text {sys }}\right) \times 10^{-12} \times(E / 1 \mathrm{TeV})^{-\gamma} \mathrm{cm}^{-2} \mathrm{~s}^{-1} \mathrm{TeV}^{-1}$ with a photon index $\gamma$ of $2.4 \pm 0.6_{\text {stat }} \pm 0.3_{\text {sys }}$, which was consistent with that derived with the ring-region background. The VHE gamma-ray extension, centroid position, and flux obtained with CANGAROO-III are consistent with results from H.E.S.S. This result suggests that the VHE gamma-ray emission was unchanged between 2004 and 2008.

\section{DISCUSSION}

We now discuss the plausible radiation mechanisms of HESS J1614-518 using the results of CANGAROO-III, H.E.S.S., Fermi, and Suzaku observations. Since the spectra of Swift sources were not available, we did not use the Swift data. Figure 5 shows the morphological relationship between each observation. The non-thermal X-ray emission from Suzaku source A is positioned very close to the H.E.S.S. gamma-ray peak, the position of the Fermi source 1FGL J1614.7-5138c, and within the emission region detected with CANGAROO-III. Thus, this could be the most likely counterpart for HESS J1614-518. We note here that since the FOV of the Suzaku observation covered only the part of the $\mathrm{TeV}$ gamma-ray emission region, as shown in Figure 5, the current observed $\mathrm{X}$-ray flux may only be a fraction of the entire $\mathrm{X}$-ray emission from the entire region of the VHE gamma-ray emission. To discuss the emission mechanism with more accuracy, further $\mathrm{X}$-ray observations of the entire region of VHE gamma-ray emission are needed. For further constraints on emission models, we tried to derive the flux at $8 \mu \mathrm{m}$ at the Suzaku source A position, from the archival data with the Infrared Array Camera (Fazio et al. 2004) on board the Spitzer Space Telescope. 


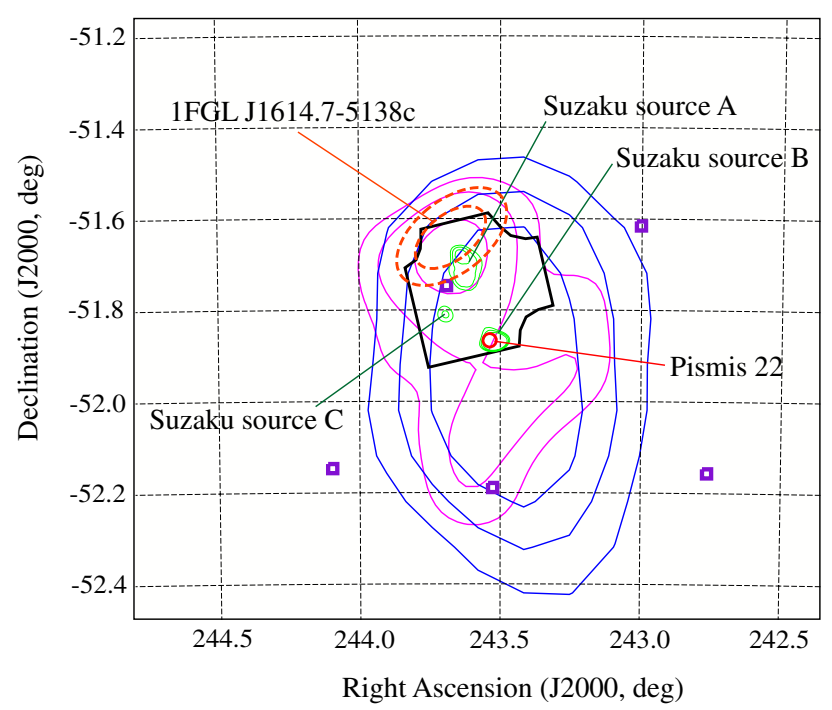

Figure 5. Morphological relationship between the X-ray and gamma-ray observations. Blue, magenta, and green contours show the emission regions of CANGAROO-III, H.E.S.S., and Suzaku, respectively. Orange dashed circles show the $68 \%$ and $90 \%$ error ellipses of the position of the Fermi source. The red circle shows the Pismis 22 position and purple squares show the positions of nearby pulsars (Manchester et al. 2005; ATNF Pulsar Catalogue ver.1.38). The bold black line shows the observed region by the Suzaku XIS in Matsumoto et al. (2008) excluding the calibration source region on the corners.

(A color version of this figure is available in the online journal.)

However, since there was contamination from a nearby source, we obtained only an upper limit.

SNR scenario. Suzaku source B, which may be related to a possible AXP, is positioned roughly in the center of the VHE gamma-ray emission. We thus postulate a scenario in which a supernova explosion occurred at the position of Suzaku source $B$ and the shock of the SNR has now reached the position of Suzaku source A, emitting both X-ray and gamma-ray emission.

$P W N$ scenario. A PWN could also emit diffuse gammaray emission. Five pulsars have been found in this region, PSR J1611-5209, PSR J1612-5136, PSR J1613-5211, PSR J1614-5144, and PSR J1616-5208 (Manchester et al. 2005; ATNF Pulsar Catalogue ver.1.38). As described in Section 1, Suzaku source B would be a PWN if associated with a pulsar or AXP. The smaller size of Suzaku source A or source B compared to that of the VHE gamma rays also appears in other PWNe because of synchrotron cooling (Section 1).

We will discuss the PWN scenario and the associated pulsar which could supply enough particles to reproduce both the X-ray emission of Suzaku source A or source B and the VHE gamma-ray emission.

Stellar wind scenario. The young open cluster Pismis 22 is located toward the center of HESS J1614-518 and is also a possible counterpart. Its age is $\sim 4.0 \times 10^{7} \mathrm{yr}$ and the distance is $1 \pm 0.4 \mathrm{kpc}$ from the Earth. The coincidence between a young open cluster and a VHE gamma-ray source is also seen in Westerlund 2 and Cyg OB2 as described in Section 1. Stellar winds from massive stars could form a shock front, accelerate charged particles, and produce high energy radiation (Voelk \& Forman 1982; Bednarek 2007).

Another possibility is an association between an SNR, PWN, and the open cluster, since Pismis 22 is old enough for some massive stars to finish their life as supernovae. In addition, as described in Section 1, binary systems that emit VHE gamma rays also have been discovered. However, HESS J1614-518 does not seem to be associated with a binary system, since all sources of this type have point-like emission, which is in contrast to the results of CANGAROO-III and H.E.S.S. We discuss the above scenarios in detail in the following subsections.

\subsection{SNR Scenario}

In the SNR scenario, we assume that the X-rays from Suzaku source $\mathrm{A}$ and the VHE gamma rays are emitted by charged particles accelerated by the shock in the SNR shell, and Suzaku source B is an associated AXP which is positioned in the center of the SNR shell. Additionally, we will discuss the possible correlation between the SNR and Pismis 22 .

First, we examine a leptonic model to explain the observed SED (Figure 6). For the X-ray spectrum, we use the Suzaku spectrum of Suzaku source A correlated with the VHE gammaray peak, with a statistical error at the $90 \%$ confidence level. For the Fermi spectrum, we used a $2 \sigma$ statistical error and systematic errors of $1.8 \sigma$ in the flux and $1.2 \sigma$ in the photon index (Abdo et al. 2010a). Figure 6 shows the simple onezone leptonic model curves for HESS J1614-518. Here we calculated synchrotron, IC, and Bremsstrahlung model curves for a single power law with an exponential cutoff electron spectrum, $d N_{e} / d E_{e}=K_{e} E_{e}^{-\Gamma_{e}} \exp \left(-E_{e} / E_{\text {max }_{e} e}\right)$, where $K_{e}$ is the normalization factor, $E_{e}$ is the electron energy, $\Gamma_{e}$ is the spectral index of the electrons, and $E_{\max _{-} e}$ is the maximum accelerated electron energy. To calculate the IC radiation, we used the cosmic microwave background and an interstellar radiation field derived from the GALPROP package (v50p) (Porter \& Strong 2005; Strong \& Moskalenko 2006) to estimate the seed photon field around the HESS J1614-518 region. Energy densities of $1.4 \mathrm{eV} \mathrm{cm}^{-3}$ and $5.5 \mathrm{eV} \mathrm{cm}^{-3}$ for IR and optical light were obtained, respectively. In addition, this radiation field changed by less than an order of magnitude when we varied the distance from $1 \mathrm{kpc}$ to $10 \mathrm{kpc}$, with values in the range from 0.9 to $1.7 \mathrm{eV} \mathrm{cm}^{-3}$ and 1.1 to $5.5 \mathrm{eV} \mathrm{cm}^{-3}$ for IR and optical light, respectively. We fixed the power-law index to $\Gamma_{e}=2.0$ and fitted the VHE gamma-ray spectrum by the IC emission. The maximum energy and total energy of the electrons obtained were $4.2 \pm 1.5 \mathrm{TeV}$ and $1.9 \times 10^{49} \times(d / 10 \mathrm{kpc})^{2} \mathrm{erg}$. From comparison between the synchrotron model and the Spitzer upper limit, an upper limit of a magnetic field is determined to be $6 \mu \mathrm{G}$. The harder and fainter spectrum in the $\mathrm{X}$-ray band compared to the VHE gamma-ray spectrum did not allow the observed X-ray and VHE gamma-ray spectra to be produced with synchrotron and IC emission, respectively, by a single power-law distribution of electrons. There is also the possibility that Bremsstrahlung produces both the X-ray and VHE gamma-ray emission (Uchiyama et al. 2002), as shown in Figure 6. This model gives a good reproduction for an ambient matter density $n_{p}$ of $600 \mathrm{p} \mathrm{cm}^{-3}$. However, Rowell et al. (2008) reported that they found no obvious overlapping molecular clouds across a range of inferred distances up to $\sim 6 \mathrm{kpc}$, with the NANTEN ${ }^{12} \mathrm{CO}(J=1-0)$ survey data (Matsunaga et al. 2001).

In addition, we estimated an ambient matter density from the velocity-integrated data of the CO survey (Dame et al. 2001) to be $\sim 80$ or $8 p \mathrm{~cm}^{-3}$ for $10 \mathrm{kpc}$ and $1 \mathrm{kpc}$, respectively. Thus, the Bremsstrahlung model that requires an ambient matter density of $600 p \mathrm{~cm}^{-3}$ was rejected.

Furthermore, there is difficulty in explaining the morphological difference between the X-ray and the VHE gamma-ray 


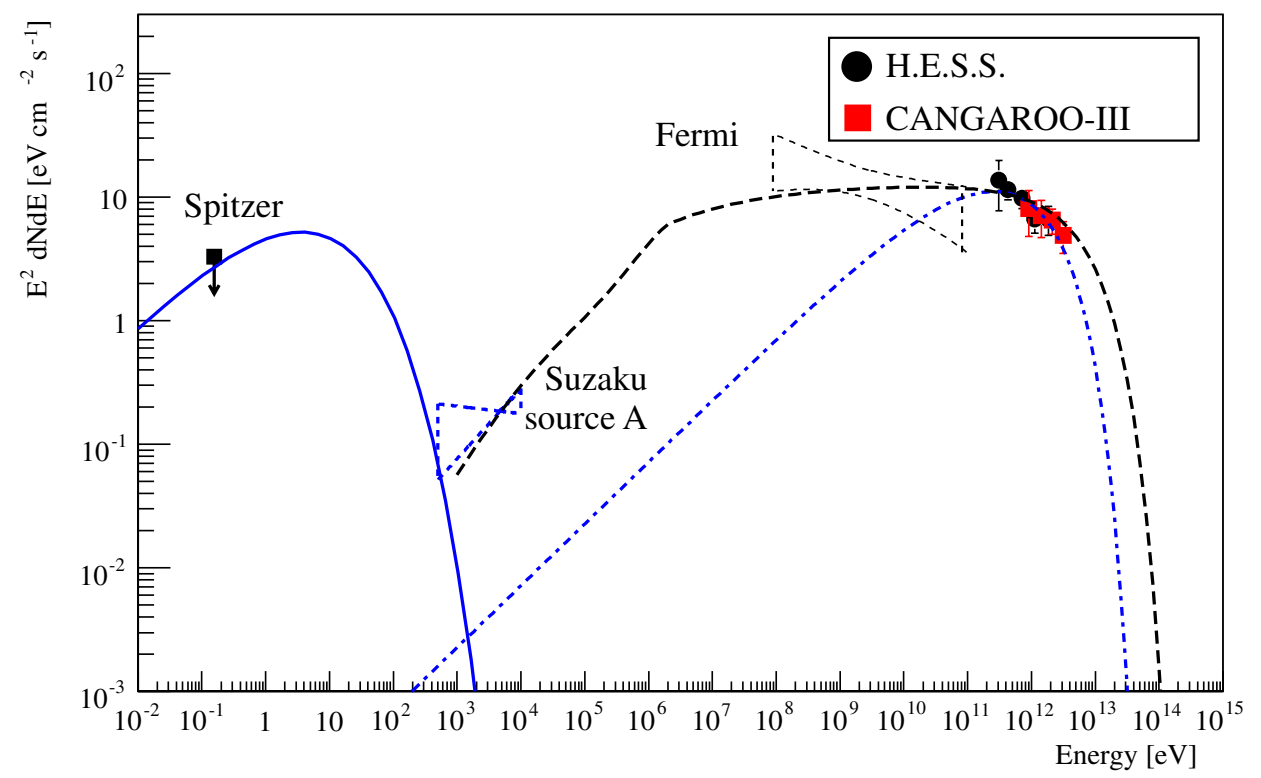

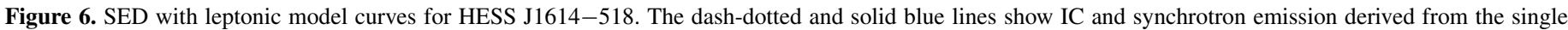

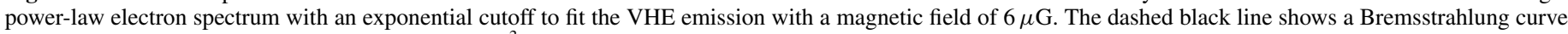
for a number density of ambient matter of $600 \mathrm{p} \mathrm{cm}^{-3}$.

(A color version of this figure is available in the online journal.)

emission because it requires an unlikely situation in which the relatively high-energy (multi-TeV) electrons that are responsible for the VHE gamma-ray emission are distributed over a more extended region than the relatively low-energy (multi-keV) electrons which are responsible for the X-ray emission.

We also checked that our estimate of the non-thermal synchrotron flux did not violate the thermal optical emission. The intrinsic optical background flux over the solid angle for the gamma-ray emission region $\sim 5 \times 10^{5} \mathrm{eV} \mathrm{cm}^{-2} \mathrm{~s}^{-1}$ is obtained from $U_{\text {opt }}(c / 4 \pi) \Omega$, where $U_{\text {opt }}$ is the energy density of $5.5 \mathrm{eV} \mathrm{cm}^{-3}$ for the optical light, $c$ is the speed of the light, and $\Omega \sim \pi(0.2 \times \pi / 180)^{2}$ sr is the solid angle for the region. The optical background radiation is $10^{5}$ times larger than the optical synchrotron flux for a magnetic field of $6 \mu \mathrm{G}$ (Figure 6).

Second, we examined a neutral-pion decay model. Based on a model proposed by Yamazaki et al. (2006), only nucleonic particles remain in an old SNR with an age of $\sim 10^{5} \mathrm{yr}$, while primary electrons have already lost most of their energy by the synchrotron cooling. Figure 7 shows the SED with the assumption that the population of accelerated protons can be expressed by a single power law with an exponential cutoff, $d N_{p} / d E_{p}=K_{p} E_{p}^{-\Gamma_{p}} \exp \left(-E_{p} / E_{\max _{-} p}\right)$. We set the power-law index to $\Gamma_{p}=2.0$. The best-fit cutoff energy was obtained to be $E_{\max _{-} p}=36 \pm 18 \mathrm{TeV}$. The total energy of high-energy protons was calculated to be $W_{p}=1.2 \times 10^{52}\left(n_{p} / 1 p \mathrm{~cm}^{-3}\right)^{-1}(\mathrm{~d} / 10 \mathrm{kpc})^{2} \mathrm{erg}$. By setting $n_{p}=100 p \mathrm{~cm}^{-3}$, the efficiency of energy conversion to accelerate protons is $10 \%$ for a typical total supernova explosion kinetic energy of $\sim 10^{51} \mathrm{erg}$. As described above, no obvious molecular cloud was found in the NANTEN ${ }^{12} \mathrm{CO}(J=1-0)$ survey data.

In addition, an ambient matter density from the velocityintegrated data of the CO survey (Dame et al. 2001) was estimated to be $\sim 80$ or $8 p \mathrm{~cm}^{-3}$ for $10 \mathrm{kpc}$ and $1 \mathrm{kpc}$, respectively. Thus, an assumption of $100 p \mathrm{~cm}^{-3}$ is likely for $10 \mathrm{kpc}$, but not for $1 \mathrm{kpc}$.
Further observations are necessary to investigate the validity of such an assumed density. Assuming the spectral index $\Gamma_{p}=\Gamma_{e}=2.0$, the maximum energy of primary electrons is determined to be $E_{\max _{-} e} \leqslant E_{\max _{-} p}$. The turnover energy of synchrotron emission is determined from $E_{\text {turn }}=22 \mathrm{keV} \times$ $\left(E_{\text {max }_{e}} / 50 \mathrm{TeV}\right)^{2} \times(B / 200 \mu \mathrm{G})$. Since the hard index of the X-ray spectrum required $E_{\text {turn }} \geqslant 10 \mathrm{keV}$, a lower limit for the magnetic field of $B \geqslant 200 \mu \mathrm{G}$ was determined from this equation. The model curve for this lower limit condition was shown in Figure 7. From this magnetic field value, we set a lower limit to the number ratio of protons to primary electrons, $K_{p e}=K_{p} / K_{e} \geqslant 2.1 \times 10^{5}\left(n_{p} / 1 \mathrm{pcm}^{-3}\right)^{-1}$.

We also calculated the contribution of emissions from secondary electrons from $p-p$ interactions between the same proton population as above and the ambient matter density of $100 p \mathrm{~cm}^{-3}$. We followed the calculation in Kelner \& Aharonian (2008) to derive the spectrum of the secondary electrons. Assuming a distance of $10 \mathrm{kpc}$ from the Earth, the distance between Suzaku source A and source B was calculated to be $35 \mathrm{pc}$. Thus, we assumed that the radius of the SNR is $35 \mathrm{pc}$ and roughly estimated the age of the SNR using the Equation (2) in Yamazaki et al. (2006) to be $3 \times 10^{4} \mathrm{yr}$. Thus, the emissions from the secondary electrons were derived by assuming continuous injection of electrons produced by a constant proton spectrum over $3 \times 10^{4} \mathrm{yr}$ with a magnetic field of $200 \mu \mathrm{G}$ (Atoyan \& Aharonian 1999). The obtained synchrotron curve is also shown in Figure 7. The obtained IC and Bremsstrahlung emissions were able to be neglected since the number of electrons is sufficiently small. Since the synchrotron emission from the secondary electrons was not able to explain the X-ray emission, the X-ray emission might originate in the synchrotron emission from the primary electrons, as shown in Figure 7, or other emission mechanisms. Since the synchrotron emission from the secondary electrons dominates below the infrared band, the detection of the emission in the radio to infrared bands could support the hypothesis that the VHE gamma-ray emission is produced by the neutral-pion decay. Because the Spitzer 


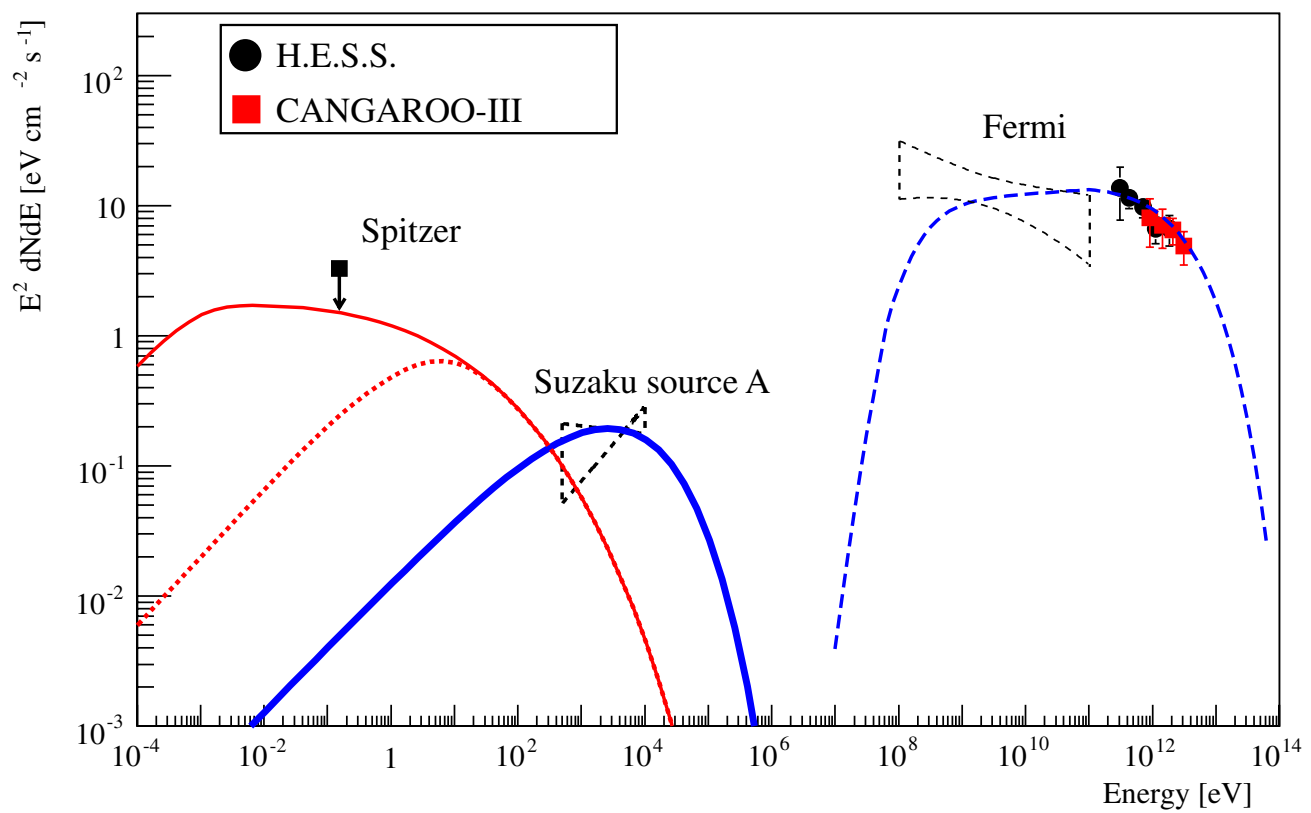

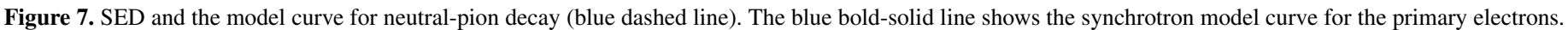
The red solid and dotted lines show synchrotron model curves for the secondary electrons for a distance of $10 \mathrm{kpc}$ and $1 \mathrm{kpc}$, respectively.

(A color version of this figure is available in the online journal.)

upper limit was above the predicted flux of synchrotron emission, more detailed observations are needed.

To discuss the possible association between the SNR and Pismis 22, we estimated the SNR age using the distance to Pismis 22 with the assumption that a supernova explosion occurred at the position of Pismis 22 and the shock front has now reached at the VHE gamma-ray peak position. By using the same Equation (2) in Yamazaki et al. (2006), the age was obtained to be $3 \times 10^{2} \mathrm{yr}$ for a distance of $1 \mathrm{kpc}$. Since the lower limit of a magnetic field was obtained to be $B \geqslant 200 \mu \mathrm{G}$ as above, the synchrotron cooling time of $100 \mathrm{TeV}$ electrons decreased to $1 \mathrm{yr}$. For a distance of $1 \mathrm{kpc}$, the required total energy of protons $W_{p}$ could be reduced if the number density of ambient matter $n_{p}$ is the same as for a distance of $10 \mathrm{kpc}$. If the total energy of protons $W_{p}$ is fixed to be $10^{50} \mathrm{erg}$, the required density of ambient matter is reduced to $n_{p}=1 p \mathrm{~cm}^{-3}$ for a distance of $1 \mathrm{kpc}$. This value was comparable with the typical number density in the interstellar field and does not contradict the fact that no obvious molecular cloud was found in the NANTEN ${ }^{12} \mathrm{CO}(J=1-0)$ survey data. The contribution of emissions from secondary electrons was shown in Figure 7, assuming the injection time of $3 \times 10^{2} \mathrm{yr}$ with a magnetic field of $200 \mu \mathrm{G}$. The synchrotron emission from the secondary electrons was not able to explain the X-ray spectrum of Suzaku source A. The $\mathrm{X}$-ray emission might originate in the synchrotron emission from the primary electrons, or from other emission mechanisms. Since the flux of synchrotron emission from the secondary electrons in the radio to infrared bands was lower than that of the case of a distance of $10 \mathrm{kpc}$, as shown in Figure 7, a determination of the spectrum below the infrared band is a key to revealing the origin of the SNR. Additionally, an observation of thin thermal plasma in the X-ray band will provide useful information such as plasma temperature or chemical abundances. For example, the detection of high abundance of $\alpha$-elements (O, Mg, Si, S, Ca, and Ti) compared to that of iron, which is expected in a massive star explosion (Kobayashi et al. 2006), may support the SNR scenario. In fact, a recent Suzaku observation of the open cluster Westerlund 2 detected metal-rich thermal emission, suggesting that the diffuse $\mathrm{X}$-ray and VHE gamma-ray emission may have originated from a hypernova remnant (Fujita et al. 2009).

\subsection{Stellar Wind Scenario}

The VHE gamma-ray emission might have been produced by hadrons accelerated in winds from massive stars in Pismis 22 (Voelk \& Forman 1982; Bednarek 2007). A fraction of the stellar wind energy can be transferred to relativistic particles. Assuming that the shock acceleration generates a single powerlaw spectrum of primary particles, we can apply the discussion made in the SNR scenario. We discuss the energetics for the hadronic origin here to produce the observed gamma-ray emission. A single O-type star loses mass at a rate of $\dot{M}=$ $10^{-6} M_{\odot}$ per year with a stellar wind velocity of $\sim 1500 \mathrm{~km} \mathrm{~s}^{-1}$ (Castor et al. 1975). The rate of kinetic energy emitted from the single star is $7 \times 10^{35} \mathrm{erg} \mathrm{s}^{-1}$. If we assume an energy conversion efficiency to particle acceleration of $5 \%$, which is the maximum efficiency adopted for a hadronic model in Bednarek (2007), an ambient matter density of $100 \mathrm{p} \mathrm{cm}^{-3}$, a distance of $1 \mathrm{kpc}$, and an age of $40 \mathrm{Myr}$, two O-type stars are required in Pismis 22 to produce the observed VHE gamma-ray spectrum in the hadronic scenario. However, no obvious molecular cloud has been found at this distance in the NANTEN data, as described above.

\subsection{PWN Scenario}

PWNe are the largest class of identified Galactic VHE gamma-ray sources. We discuss the possibility of HESS J1614-518 being a PWN in this subsection.

As calculated in Rowell et al. (2008), the spin-down luminosity of each of the five nearby pulsars (Figure 5) is smaller than the TeV gamma-ray luminosity. Thus, none of already known pulsars can be associated with HESS J1614-518. There is also the possibility that an undiscovered pulsar with a high spin-down power, sufficient to explain the observed gamma-ray 
luminosity, might be located in the vicinity of Suzaku source A or source B. We estimated the pulsar age to be $24 \mathrm{kyr}$ and $23 \mathrm{kyr}$ for Suzaku source A and B, respectively, using the correlation between pulsar age and the ratio of gamma-ray flux and X-ray flux from the PWN (Mattana et al. 2009). For this relatively old age, we should apply the time-evolving electron injection model (Section 1). We applied this model to HESS J1614-518, following the calculation in Higashi et al. (2008) applied to HESS J1804-216. Although this model can explain the large ratio between the X-ray and TeV gamma-ray fluxes assuming a single power-law electron distribution, the model showed a very large discrepancy with the sub-GeV flux observed with Fermi; the model curve is 30 times larger than the observed flux at $0.1 \mathrm{GeV}$. Thus, the time-evolving electron injection model with a single power-law electron distribution was rejected. The $\mathrm{MeV} /$ $\mathrm{GeV}$ component could arise from the different mechanism than the TeV emission.

Although the present sensitivity in the radio band may not be sufficient to detect this unknown pulsar, further observations in the $\mathrm{GeV}$ band could detect a radio-quiet pulsar like Geminga, which was detected with CGRO EGRET (Bertsch et al. 1992), or 16 previously unknown pulsars which were recently discovered with Fermi (Abdo et al. 2009). In addition, future X-ray observations could detect pulsed emission from Suzaku source A or source B. Given these detections, emission models for PWNe (e.g., Abdo et al. 2010b; Tanaka \& Takahara 2010; Slane et al. 2010; Bucciantini et al. 2011), using a broken power-law distribution of electrons, might be able to reveal that the VHE gamma-ray emission of this unidentified source originates from a PWN.

\section{CONCLUSION}

The observation of HESS J1614-518 with the CANGAROOIII telescopes confirms the VHE gamma-ray emission reported by H.E.S.S. The differential energy spectrum can be fitted with a single power law: $\left(8.2 \pm 2.2_{\text {stat }} \pm 2.5_{\text {sys }}\right) \times 10^{-12} \times(E / 1$ $\mathrm{TeV})^{-\gamma} \mathrm{cm}^{-2} \mathrm{~s}^{-1} \mathrm{TeV}^{-1}$ with a photon index $\gamma$ of $2.4 \pm$ $0.3_{\text {stat }} \pm 0.2_{\text {sys. }}$. We discuss the possible counterparts for this object using the results of observations with Suzaku and Fermi. For the SNR scenario, a one-zone leptonic model was not able to account for the observed SED. Hadronic models gave a good reproduction of the SED and the typical SNR explosion energy of $\sim 10^{51} \mathrm{erg}$ is able to supply the total energy of protons. Since the required number densities of the ambient matter were $n_{p}=$ $1 p \mathrm{~cm}^{-3}$ and $n_{p}=100 p \mathrm{~cm}^{-3}$ for a distance to the SNR of $1 \mathrm{kpc}$ and $10 \mathrm{kpc}$, respectively, detailed molecular observations could determine whether the SNR originated from Pismis 22 (d $\sim 1 \mathrm{kpc}$ ) or a farther distance. As there were also differences in the spectrum of the emission from the secondary electrons, a determination of the spectrum below the infrared band would help determine the likelihood of an SNR origin. For the PWN scenario, the nearby known pulsars are not responsible since the spin-down powers are insufficient to produce the observed $\mathrm{TeV}$ gamma-ray luminosity. Further observations to search a pulsar are necessary to investigate the PWN scenario. For the stellar wind scenario, Pismis 22 was required to contain two O-type stars through its entire age from energetics considerations. However, the required number density of the ambient matter of $n_{p}=100 p \mathrm{~cm}^{-3}$ may not be consistent with the results of the NANTEN observations.

To identify HESS J1614-518, more detailed multiwavelength observations are required. To discuss the stellar wind origin in more detail, a determination of the number of OB stars is necessary. For the SNR scenario, the ultra-high-energy resolution of the soft X-ray spectrometer on board Astro- $H$ could detect line emissions with a high abundance of $\alpha$-elements compared to that of iron, which would indicate that HESS J1614-518 is an SNR. To show that the Suzaku source B is an AXP, supporting the SNR or PWN scenario, a high time-resolution X-ray observation is needed to detect a pulsed signal from the source. More detailed gamma-ray spectroscopy with Fermi and the Cherenkov Telescope Array (CTA 2010) could determine the origin of the accelerated particles.

The authors thank H. Matsumoto for providing useful information about the Suzaku data, H. Yamamoto for fruitful discussion about the CO data, and S. Nishiyama and T. Sawano for helping us use the Spitzer data. We also thank the anonymous referee for helpful comments. This work was supported by a Grant-in-Aid for Scientific Research by the Japan Ministry of Education, Culture, Sports, Science and Technology (MEXT), the Australian Research Council, and the Inter-University Research Program of the Institute for Cosmic Ray Research. The work is also supported by Grant-in-Aid for the Global COE Program "The Next Generation of Physics, Spun from Universality and Emergence" from MEXT of Japan. We thank the Defence Support Center Woomera and BAE systems and acknowledge all the developers and collaborators on the GALPROP project. T. Mizukami was supported by Japan Society for the Promotion of Science Research Fellowships for Young Scientists.

\section{REFERENCES}

Abdo, A. A., Ackermann, M., Ajello, M., et al. 2010a, ApJS, 188, 405 Abdo, A. A., Ackermann, M., Ajello, M., et al. 2010b, ApJ, 714, 927 Abdo, A. A., Ackermann, M., Ajello, M., et al. 2009, ApJS, 187, 460 Abdo, A. A., Allen, B., Berley, D., et al. 2007, ApJ, 664, L91

Aharonian, F., Akhperjanian, A., Beilicke, M., et al. 2002, A\&A, 393, L37 Aharonian, F., Akhperjanian, A., Beilicke, M., et al. 2004, ApJ, 614, 897 Aharonian, F., Akhperjanian, A., Beilicke, M., et al. 2005a, A\&A, 431, 197 Aharonian, F., Akhperjanian, A. G., Aye, K.-M., et al. 2005b, A\&A, 435, L17 Aharonian, F., Akhperjanian, A. G., Aye, K.-M., et al. 2005c, Science, 307, 1938

Aharonian, F., Akhperjanian, A. G., Aye, K.-M., et al. 2005d, A\&A, 442, 1

Aharonian, F., Akhperjanian, A. G., Barres de Almeida, U., et al. 2008, A\&A, 477,353

Aharonian, F., Akhperjanian, A. G., Bazer-Bachi, A. R., et al. 2005e, A\&A, 437, L7

Aharonian, F., Akhperjanian, A. G., Bazer-Bachi, A. R., et al. 2006a, ApJ, 636, 777

Aharonian, F., Akhperjanian, A. G., Bazer-Bachi, A. R., et al. 2006b, A\&A, 448, L43

Aharonian, F., Akhperjanian, A. G., Bazer-Bachi, A. R., et al. 2006c, A\&A, 449, 223

Aharonian, F., Akhperjanian, A. G., Bazer-Bachi, A. R., et al. 2006d, A\&A, 460,365

Aharonian, F., Akhperjanian, A. G., Bazer-Bachi, A. R., et al. 2007, A\&A, 467, 1075

Aharonian, F., Akhperjanian, A. G., de Almeida, U. B., et al. 2009, ApJ, 692, 1500

Albert, J., Aliu, E., Anderhub, H., et al. 2006, Science, 312, 1771

ATNF Pulsar Catalogue, http://www.atnf.csiro.au/research/pulsar/psrcat/

Atoyan, A. M., \& Aharonian, F. A. 1999, MNRAS, 302, 253

Bednarek, W. 2007, MNRAS, 382, 367

Bertsch, D. L., Brazier, K. T. S., Fichtel, C. E., et al. 1992, Nature, 357, 306

Bock, D. C.-J., Large, M. I., \& Sadler, E. M. 1999, AJ, 117, 1578

Bucciantini, N., Arons, J., \& Amato, E. 2011, MNRAS, 410, 381

Castor, J. I., David, C., \& Klein, R. I. 1975, ApJ, 195, 157

CTA Consortium 2010, arXiv:1008.3703

Dame, T. M., Hartmann, D., \& Thaddeus, P. 2001, ApJ, 547, 792

Dickey, J. M., \& Lockman, F. J. 1990, ARA\&A, 28, 215

Dodson, R., Legge, D., Reynolds, J. E., \& McCulloch, P. M. 2003, ApJ, 596, 1137

Enomoto, R., Hara, S., Asahara, A., et al. 2002a, Astropart. Phys., 16, 235 
Enomoto, R., Kushida, J., Nakamori, T., et al. 2009, ApJ, 703, 1725 Enomoto, R., Tanimori, T., Naito, T., et al. 2002b, Nature, 416, 823 Enomoto, R., Tsuchiya, K., Adachi, Y., et al. 2006a, ApJ, 638, 397

Enomoto, R., Watanabe, S., Tanimori, T., et al. 2006b, ApJ, 652, 1268

Fazio, G. G., Hora, J. L., Allen, L. E., et al. 2004, ApJS, 154, 10

Fahlman, G. G., \& Gregory, P. C. 1981, Nature, 293, 202

Fisher, R. A. 1936, Ann. Eugenics, 7, 179

Fujita, Y., Hayashida, K., Takahashi, H., \& Takahara, F. 2009, PASJ, 61, 1229

Funk, S., Hinton, J. A., Pühlhofer, G., et al. 2007, ApJ, 662, 517

Gaensler, B. M., Arons, J., Kaspi, V. M., et al. 2002, ApJ, 569, 878

Gaensler, B. M., Brazier, K. T. S., Manchester, R. N., Johnston, S., \& Green, A. J. 1999, MNRAS, 305, 724

Higashi, Y., Kubo, H., Yoshida, T., et al. 2008, ApJ, 683, 957

Hillas, A. M. 1985, in Proc. 19th ICRC (La Jolla), Vol. 3, 445

Kabuki, S., Enomoto, R., Bicknell, G. V., et al. 2007, ApJ, 668, 968

Kabuki, S., Tsuchiya, K., Okumura, K., et al. 2003, Nucl. Instrum. Methods Phys. Res. A, 500, 318

Katagiri, H., Enomoto, R., Ksenofontov, L. T., et al. 2005, ApJ, 619, L163

Kawachi, A., Hayami, Y., Jimbo, J., et al. 2001, Astropart. Phys., 14, 261

Kelner, S. R., \& Aharonian, F. A. 2008, Phys. Rev. D, 78, 034013

Kobayashi, C., Umeda, H., Nomoto, K., Tominaga, N., \& Ohkubo, T. 2006, ApJ, 653,1145

Kubo, H., et al. 2001, in Proc. 27th ICRC (Hamburg), 2900

Kuiper, L., Hermsen, W., den Hartog, P. R., \& Collmar, W. 2006, ApJ, 645, 556

Landi, R., Bassani, L., Malizia, A., et al. 2006, ApJ, 651, 190

Landi, R., Masetti, N., Bassani, L., et al. 2007, ATel, 1047

Malkov, M. A., Diamond, P. H., \& Sagdeev, R. Z. 2005, ApJ, 624, L37

Manchester, R. N., Hobbs, G. B., Teoh, A., \& Hobbs, M. 2005, AJ, 129, 1993

Manzali, A., De Luca, A., \& Caraveo, P. A. 2007, ApJ, 669, 570

Markwardt, C. B., \& Ogelman, H. 1995, Nature, 375, 40

Matsumoto, H., et al. 2008, PASJ, 60, 123

Matsunaga, K., Mizuno, N., Moriguchi, Y., et al. 2001, PASJ, 53, 1003
Mattana, F., Falanga, M., Götz, D., et al. 2009, ApJ, 694, 12

Muno, M. P., Law, C., Clark, J. S., et al. 2006, ApJ, 650, 203

Muraishi, H., Tanimori, T., Yanagita, S., et al. 2000, A\&A, 354, L57

Murphy, T., Mauch, T., Green, A., et al. 2007, VizieR Online Data Catalog, 8082,0

Nakamori, T., Kubo, H., Yoshida, T., et al. 2008, ApJ, 677, 297

Ohm, S., et al. 2009, arXiv:0906.2637

Ohm, S., et al. 2010, in ASP Conf. Ser. 422, High Energy Phenomena in Massive Stars, ed. J. Martí, P. L. Luque-Escamilla, \& J. A. Combi (San Francisco, CA: ASP), 265

Piatti, A. E., Claria, J. J., \& Bica, E. 2000, A\&A, 360, 529

Porter, T. A., \& Strong, A. W. 2005, in Proc. 29th ICRC (Pune), Vol., 4, 77

Rowell, G., Horns, D., Fukui, Y., \& Moriguchi, Y. 2008, in AIP Conf. Ser. 1085, High Energy Gamma-ray Astronomy, (Melville, NY: AIP), 241

Slane, P., Castro, D., Funk, S., et al. 2010, ApJ, 720, 266

Strong, W. S., \& Moskalenko, I. V. 2006, GALPROP Project, http://galprop.stanford.edu/web_galprop/galprop_home.html

Sturner, S. J., Skibo, J. G., Dermer, C. D., \& Mattox, J. R. 1997, ApJ, 490, 619

Tanaka, S. J., \& Takahara, F. 2010, ApJ, 715, 1248

Tanaka, T., Uchiyama, Y., Aharonian, F. A., et al. 2008, ApJ, 685, 988

TeVCAT http://tevcat.uchicago.edu/

Uchiyama, Y., Aharonian, F. A., Tanaka, T., Takahashi, T., \& Maeda, Y. 2007, Nature, 449, 576

Uchiyama, H., Matsumoto, H., Tsuru, T. G., Koyama, K., \& Bamba, A. 2009, PASJ, 61, 189

Uchiyama, Y., Takahashi, T., Aharonian, F. A., \& Mattox, J. R. 2002, ApJ, 571, 866

Vasisht, G., \& Gotthelf, E. V. 1997, ApJ, 486, L129

Voelk, H. J., \& Forman, M. 1982, ApJ, 253, 188

Watanabe, S., et al. 2003, in Proc. 28th ICRC (Tsukuba), Vol. 4, 2397

Weekes, T. C., Cawley, M. F., Fegan, D. J., et al. 1989, ApJ, 342, 379

Willingale, R., Aschenbach, B., Griffiths, R. G., et al. 2001, A\&A, 365, L212

Yamazaki, R., Kohri, K., Bamba, A., et al. 2006, MNRAS, 371, 1975

Yamazaki, R., Kohri, K., \& Katagiri, H. 2009, A\&A, 495, 9 